\title{
Dialisi peritoneale in Italia: adeguatamente utilizzata o è possibile un ulteriore incremento?
}

\author{
Giuliano Brunori
}

\author{
Cattedra e Divisione di Nefrologia e Dialisi \\ Università e Spedali Civili, Brescia
}

\section{A}

partire dagli anni ' $80 \mathrm{nu}$ merose sono state le pubblicazioni del gruppo di Brescia che hanno evidenziato come con la dialisi peritoneale fosse possibile ottenere nei pazienti uremici una sopravvivenza sovrapponibile a quella osservata nei pazienti trattati con dialisi extracorporea. Negli anni '90, invece, l'attenzione del nostro gruppo è stata rivolta prevalentemente alla ricerca della dose dialitica necessaria a definire la quantità di dialisi per ottenere un trattamento dialitico adeguato.

Alcuni anni dopo le DOQI Guidelines per il trattamento peritoneale indicavano come parametro per l'adeguatezza dialitica il valore da noi riportato e che, successivamente, era stato confermato anche dal Canusa Study.

Dopo anni di costante incremento del numero di pazienti, che ci ha permesso di ottenere un'esperienza notevole nel trattamento con dialisi peritoneale, anche il nostro gruppo ha assistito a un costante decremento dei pazienti che sceglievano questa metodica per il trattamento dialitico. Alla fine del 2000 avevamo in trattamento circa 70 pazienti, a fronte di oltre 120 pazienti nel corso del 1992.

Cosa può essere cambiato in pochi anni nella nostra popolazione da rendere più difficile la scelta della DP? E quali possono essere state le ragio- ni per cui altre zone del Paese hanno visto una scarsa, se non assente, presenza della dialisi peritoneale fra le opzioni per il trattamento dialitico sostitutivo?

A mio giudizio, maturato anche con lo scambio di opinioni fra i colleghi con cui lavoro giornalmente o con quanti, per altri motivi, ho la possibilità di confrontarmi, diversi sono i fattori che possono aver contribuito al ridotto sviluppo della dialisi peritoneale.

All'inizio della sua diffusione la dialisi peritoneale era gravata da un alto numero di insuccessi, in particolare dovuto all'incidenza di infezioni, per cui tale metodica doveva essere utilizzata solo per pazienti ormai non più suscettibili di un trattamento extracorporeo o con una scarsa attesa di vita. L'articolo pubblicato dal prof. Shaldon in cui definiva la CAPD come una seconda scelta, per pazienti di seconda scelta e per medici di seconda scelta non passò inosservato. Molti trassero e forse ancora oggi, purtroppo, traggono da questa pubblicazione il convincimento nel rifiuto dell'utilizzo della dialisi peritoneale. In quanti convegni abbiamo sentito illustri colleghi scagliarsi ancora contro la DP utilizzando questi dati!

Ma quello che maggiormente mi sconcerta è che le numerose pubblicazioni che negli anni successivi han- no dimostrato il calo delle complicanze infettive, la non diversa sopravvivenza dei pazienti nelle due metodiche (DP e ED), la buona riabilitazione dei pazienti, in particolare gli anziani, i buoni risultati ottenuti nei pazienti che venivano successivamente trapiantati, il minor rischio di contrarre epatite, la miglior stabilità cardiovascolare non hanno avuto su molti dei nefrologi italiani alcun effetto.

Probabilmente una maggior attenzione a quanto veniva riportato da quanti giornalmente si confrontavano con questa metodica, doveva permettere una diffusione più capillare su tutto il territorio nazionale.

La necessità del catetere peritoneale (e quindi i connessi rischi infettivi) e l'uso di una membrana biologica (e quindi il suo possibile esaurimento nel tempo) sono due altri punti su cui, in molti, hanno costruito le loro "certezze" nel rifiutare questa metodica. All'ultimo Corso di aggiornamento-San Carlo, il prof. Cancarini ha illustrato, con dovizia di dati, come la peritonite sclerosante, complicanza più temibile, interessa meno del $4 \%$ dei pazienti trattati, mentre sempre più sono le pubblicazioni che riportano l'incidenza di peritonite a 1 episodio ogni 30-36 mesi/paziente. Anche questi dati dovrebbero ridurre sempre più il numero dei nefrologi "scettici" o "avversi" alla DP. 
La dialisi peritoneale, come ogni altra metodica domiciliare, deve basarsi su una corretta informazione del paziente, che deve essere effettuata già nella fase del trattamento conservativo e non nelle giornate precedenti l'inizio del trattamento. Un paziente ben informato sceglierà la metodica che meno interferisce con il suo vivere quotidiano, e la scelta del trattamento sarà ponderata e convinta.

Durante il recente congresso della SIN a Taormina, il Gruppo di Studio per la Dialisi Peritoneale ha proposto un questionario (purtroppo compilato da meno di 200 nefrologi), e tra le indicazioni alla dialisi peritoneale la volontà del paziente ha ricevuto scarsa attenzione, anche se costituisce il cardine di questo trattamento domiciliare. Quanti sono nei vari Centri italiani i pazienti che non hanno potuto scegliere il trattamento? Quanti sono stati avviati alla peritoneale solo per mancanza dei posti tecnici per l'extracorporea e quanti, sicuramente in numero maggiore, non hanno potuto scegliere ma gli è stato imposto il trattamento che si era già scelto?

Sulla scorta di queste mie riflessioni ritengo necessario, pertanto, che in ogni ambulatorio nefrologico il paziente con insufficienza renale debba essere informato, per tempo, sulle possibilità di trattamento, sui vantaggi e svantaggi che le metodiche hanno, sulla necessità che per determinate condizioni cliniche un trattamento sia più adeguato di un altro. Al termine di questo "percorso" il paziente troverà le giuste indicazioni per scegliere la metodica a lui più indicata. Un altro argomento di discussione sono i rimborsi. Diverse analisi dei costi del trattamento hanno evidenziato come la dialisi peritoneale ambulatoriale continua abbia un costo inferiore anche del $30 \%$ rispetto al trattamento in extracorporea. Tuttavia, anche queste valutazioni hanno avuto un basso "peso" sull'indicazione al trattamento dialitico. In epoca di contrazione della spesa sanitaria, di budget da rispettare, di possibili tagli alle unità operative per eccesso di spesa, l'implementazione dell'uso della DP potrebbe permettere un contenimento dei costi generali, permet- tendo così il trattamento di un numero più elevato di pazienti.

Infine, non va dimenticato come in questi anni, in alcune aree del Paese, abbiamo assistito a una modifica del nucleo familiare, con anziani sempre più soli e senza il supporto necessario di partner per la gestione del trattamento dialitico. Soprattutto nelle grandi città perché non istituire un programma di assistenza domiciliare per il trattamento dialitico o creare Centri dove effettuare dialisi automatizzata durante le ore notturne (utilizzo delle residenze sanitarie, centri sociali per anziani) gestita o dall'unità sanitaria o direttamente dalle ditte produttrici?

Sulla scorta di quanto finora riportato ritengo pertanto che la dialisi peritoneale abbia, ancora oggi, un enorme spazio di crescita e ciò può avvenire se questa metodica viene vista non antagonista al trattamento extracorporeo ma come una delle opzioni possibili del trattamento dell'uremia terminale.

In conclusione, per stimolare discussione e quindi approfondimento del problema, suggerirei alcuni punti per migliorare l'utilizzo e lo sviluppo della dialisi peritoneale:

1) Utilizzare la dialisi peritoneale come prima scelta (nei pazienti in cui non sussistono controindicazioni) al fine di "sfruttare" il contributo dato nella fase iniziale del trattamento dalla funzione renale residua.

2) Cercare di mantenere la diuresi residua, anche a costo di una modesta iperidratazione, evitando in particolare l'uso di farmaci nefrotossici.

3) Stabilire, a livello nazionale, la percentuale di pazienti in trattamento dialitico peritoneale per ogni Centro dialisi. Il raggiungimento di questo target dovrebbe garantire la disponibilità di fondi per il Centro (a esempio la differenza ottenuta se tutti i pazienti fossero in trattamento extracorporeo dovrebbe costituire un fondo comune per gli operatori del Centro).

4) Favorire l'addestramento, anche a domicilio dei pazienti da parte delle ditte fornitrici del materiale.
5) Creare sul territorio una rete di Centri in cui effettuare la dialisi automatizzata notturna per pazienti non in grado di auto-gestirsi o sprovvisti di partner.

6) Garantire ai pazienti che effettuano un trattamento domiciliare un assegno mensile tipo "assegno di accompagnamento”.

gcbrunori@hotmail.com 